\title{
BMJ Open Systematic review of the global epidemiology of viral-induced acute liver failure
}

\author{
Jenna Patterson (10 , , Hannah Sophia Hussey, ${ }^{1}$ Sheetal Silal, ${ }^{2,3}$ Liz Goddard, ${ }^{4}$ \\ Mashiko Setshedi, ${ }^{5}$ Wendy Spearman, ${ }^{6}$ Gregory D Hussey, ${ }^{1,7}$ Benjamin M Kagina, ${ }^{1}$ \\ Rudzani Muloiwa ${ }^{1,8}$
}

\begin{abstract}
To cite: Patterson J, Hussey HS, Silal S, et al. Systematic review of the global epidemiology of viral-induced acute liver failure. BMJ Open 2020;10:e037473. doi:10.1136/ bmjopen-2020-037473

- Prepublication history and additional material for this paper are available online. To view these files, please visit the journal online (http://dx.doi. org/10.1136/bmjopen-2020037473).
\end{abstract}

Received 07 February 2020

Revised 04 June 2020

Accepted 04 June 2020

\section{ABSTRACT}

Objectives The aetiology and burden of viral-induced acute liver failure remains unclear globally. It is important to understand the epidemiology of viral-induced ALF to plan for clinical case management and case prevention. Participants This systematic review was conducted to synthesize data on the relative contribution of different viruses to the aetiology of viral-induced acute liver failure in an attempt to compile evidence that is currently missing in the field. EBSCOhost, PubMed, ScienceDirect, Scopus and Web of Science were searched for relevant literature published from 2009 to 2019. The initial search was run on 9 April 2019 and updated via PubMed on 30 September 2019 with no new eligible studies to include. Twenty-five eligible studies were included in the results of this review. Results This systematic review estimated the burden of acute liver failure after infection with hepatitis B virus, hepatitis $A$ virus, hepatitis $C$ virus, hepatitis $E$ virus, herpes simplex virus/human herpesvirus, cytomegalovirus, Epstein-Barr virus and parvovirus B19. Data were largely missing for acute liver failure after infection with varicellazostervirus, human parainfluenza viruses, yellow fever virus, coxsackievirus and/or adenovirus. The prevalence of hepatitis A-induced acute liver failur was markedly lower in countries with routine hepatitis $A$ immunisation versus no routine hepatitis $A$ immunisation. Hepatitis $E$ virus was the most common aetiological cause of viral-induced acute liver failure reported in this review. In addition, viralinduced acute liver failure had poor outcomes as indicated by high fatality rates, which appear to increase with poor economic status of the studied countries.

Conclusions Immunisation against hepatitis $A$ and hepatitis $B$ should be prioritised in low-income and middle-income countries to prevent high viral-induced acute liver failure mortality rates, especially in settings where resources for managing acute liver failure are lacking. The expanded use of hepatitis $\mathrm{E}$ immunisation should be explored as hepatitis E virus was the most common cause of acute liver failure.

Registration PROSPERO registration number: CRD42017079730. and permissions. Published by BMJ.

For numbered affiliations see end of article.

Correspondence to Jenna Patterson; PTTJEN005@myuct.ac.za

\section{Strengths and limitations of this study}

- Comprehensive and exhaustive search for relevant studies from several databases.

- Comprehensive diagnostic inclusion criteria for acute liver failure cases according to international guideline.

- Lack of language restrictions in search led to inclusion of geographically diverse data.

- Findings limited by lack of data for some of the viral aetiologies of acute liver failure which may have led to an underestimation of the global burden of viralinduced acute liver failure.

- Diversity of viruses attributable to acute liver failure cases and viral detection methods led to high heterogeneity and low statistical power in metaanalyses conducted.

in an individual without pre-existing liver disease. ${ }^{1}$ The presence of encephalopathy is not required to define ALF in paediatric cases, but is an essential component of the definition of ALF in adults. ${ }^{1}$ Possible causes of ALF include viral infections, drugs and toxins, pregnancy-related liver diseases, vascular causes and/or malignancies. Acute viral hepatitis has been identified as the most common cause of ALF among all ages in Asia and Africa and one of the most common causes of ALF in children in Asia and South America. ${ }^{12}$ The incidence of viral-induced ALF has substantially declined in Europe after the introduction of universal immunisation against the hepatitis B virus (HBV), with only $19 \%$ of all ALF cases now attributable to viral infection in the European population. ${ }^{3}$ The introduction of routine immunisation against the hepatitis A virus (HAV) in Argentina has reduced the number of hepatitis A-induced ALF cases by more than $25 \% .^{3}$

Fatality rates associated with ALF vary between $60 \%$ and $80 \%$ depending on the disease aetiology as well as a patient's access 
to care. ${ }^{45}$ Liver transplantation plays a central role in the management of ALF and remains the only definitive treatment for patients who fail to demonstrate spontaneous recovery. ${ }^{6}$ A large proportion of patients with ALF in both high and low resource settings, however, are deemed to have contraindications to transplantation or deteriorate beyond transplantation before a liver donor is found. ${ }^{7-9}$

The burden of viral-induced ALF around the world still remains unclear, with little to no data collected regarding the disease incidence. ${ }^{1}$ Establishing the aetiology of viral-induced ALF is important for early initiation of treatment, determining the prognosis of the liver failure and identifying potential contraindications to liver transplantation. Most importantly, understanding the epidemiology of vaccine-preventable aetiologies of ALF should be prioritised in under-resourced regions with limited access to facilities for transplantation. This review aims to synthesise data on the relative contribution of different viruses to the aetiology of viral-induced ALF in an attempt to compile evidence that is currently missing in the field.

Bernal et al. completed a review of the burden of acute liver failure based on literature published between 1997 and 2009. The review became the bases for guidelines for clinical practice. ${ }^{4}$ In this systematic review, we assess whether data have changed following the Bernal et al. publication, and whether there is evidence to warrant a review of clinical practice.

\section{Objectives}

- To estimate the prevalence of hepatitis A virus (HAV), hepatitis B virus (HBV), hepatitis C virus (HCV), hepatitis D virus (HDV), hepatitis E virus (HEV), EpsteinBarr virus (EBV), herpes simplex virus-1 (HSV1), herpes simplex virus-2 (HSV2), varicella-zoster virus (VZV), parvovirus B19, human parainfluenza viruses (HPIVs), yellow fever virus (YFV), human herpesvirus 6 (HHV-6), cytomegalovirus (CMV), coxsackievirus (CA16) and/or adenovirus (HAdVs) among patients with ALF.

- To estimate the mortality rate for cases of ALF after infection with HAV, HBV, HCV, HDV, HEV, EBV, HSV1, HSV2, VZV, parvovirus B19, HPIVs, YFV, HHV-6, CMV, CA16 and/or HAdVs.

- To estimate the prevalence and incidence of liver transplantation for cases of ALF after infection with HAV, HBV, HCV, HDV, HEV, EBV, HSV1, HSV2, VZV, parvo-virus B19, HPIVs, YFV, HHV-6, CMV, CA16 and/ or HAdVs.

\section{METHODS}

This systematic review was registered with PROSPERO (registration number CRD42017079730) and the review methods have been published. ${ }^{10}$ The results of the review are reported using the Preferred Reporting Items for Systematic Reviews and Meta-analyses guidelines checklist.

\section{Study eligibility criteria}

Published cross-sectional, surveillance and cohort studies reporting the outcomes of interest in patients with ALF after infection with HAV, HBV, HCV, HDV, HEV, EBV, HSV1, HSV2, VZV, parvovirus B19, HPIVs, YFV, HHV-6, CMV, CA16 and/or HAdVs were eligible for inclusion in this study. Studies were eligible for inclusion if they had clearly stated case definitions of viral-induced ALF and confirmed ALF cases using both clinical and serological, molecular or culture diagnostic methods.

\section{Search strategy}

A combination of the following search terms (including the use of Medical Subject Headings (MESH)) was used and adapted for each of the relevant electronic databases: epidemiology, prevalence, incidence, burden, mortality, morbidity, fulminant hepatic failure, fulminant liver failure, acute hepatic failure, acute liver failure, hepatitis A virus (HAV), hepatitis B virus ( $\mathrm{HBV}$ ), hepatitis $\mathrm{C}$ virus (HCV), hepatitis D virus (HDV), hepatitis E virus (HEV), Epstein Barr virus (EBV), herpes simplex virus-1 (HSV1), herpes simplex virus-2 (HSV2), varicella-zoster virus (VZV), parvo-virus B19, human parainfluenza viruses (HPIVs), yellow fever virus (YFV), human herpesvirus 6 (HHV-6), cytomegalovirus (CMV), coxsackievirus and adenovirus.

The following electronic databases were searched for relevant literature published from 2009 to 2019: EBSCOhost, PubMed, ScienceDirect, Scopus and Web of Science. The search was run on 9 April 2019 and updated via PubMed on 30 September 2019 with no new eligible studies to include.

\section{Data extraction}

Study characteristics and outcomes of interests were extracted from the included studies on a predesigned data extraction form by two independent reviewers (JP and $\mathrm{HSH}$ ). Prior to use by the two reviewers, the reliability of the extraction form was assessed by piloting 10 randomly selected articles that met the inclusion criteria. The study team resolved any disagreements in data extraction through consensus in consultation with RM. In cases where studies were in German, HSH provided translation. In cases where studies were not available in English or German, Google translate was used to translate the article to English. ${ }^{11}$

\section{Data synthesis and analysis}

A random-effects model was fitted to the study data as it included data taken from a series of independently performed studies in different populations. We assessed heterogeneity by calculating $\mathrm{I}^{2}$ statistics (threshold $\left.\mathrm{I}^{2}>40 \%\right)$. The values of $\mathrm{I}^{2}$ were categorised for heterogeneity as follows: 'not important' $(\leq 40 \%)$, 'moderate' (> $40 \%$ to $\leq 60 \%)$ and 'considerable' $(>60 \%$ to $\leq 80 \%)$ and 'substantial' ( $>80 \%$ to $\leq 100 \%)$. Where 'not important' or 'moderate' heterogeneity existed between studies $\left(\mathrm{I}^{2} \leq 60 \%\right)$, pooled outcome measures were reported with 
95\% CI for each respective outcome. Where 'considerable' or 'substantial' heterogeneity exists between studies $\left(\mathrm{I}^{2}>60 \%\right)$, forest plots and prevalence ranges calculated using the random-effects model were used to narratively describe each outcome.

\section{Risk of bias assessment}

Each included study was assessed for risk of bias and quality using the Hoy et al tool for observational studies. ${ }^{12} 13$ Studies were judged as having 'low risk' if scored 8-10, 'moderate risk' if scored 5-7 and 'high risk' if scored 0-5. All risk of bias judgements were made by both JP and $\mathrm{HSH}$. In case of disagreement in risk of bias and quality assessment, a final decision was made through consensus in consultation with RM.

\section{Patient and public involvement}

This review was developed as part of an ongoing project by the research team that aims to generate evidence to facilitate evidence-based decision-making of introducing routine hepatitis A vaccination in South Africa. The findings of this review contribute to the knowledge base that aims to enhance global vaccination strategies against viral-associated ALF. As this is a systematic review, no patient involvement was required; however, it is hoped that the findings of this review will help to highlight the burden that ALF places on populations without routine vaccination.

\section{RESULTS}

The initial database searches yielded 6952 records, from which 3545 duplicates were removed. A further 3263 were excluded following the screening of titles and abstracts (figure 1). The full text of the remaining 144 records were screened by JP and $\mathrm{HSH}$, from which 25 studies were deemed to meet the final inclusion criteria. Twenty-four $(96 \%)$ of the included studies were cohort studies. As detailed in table 1, the included studies were published between 2009 and 2017. Included studies were conducted globally, with seven studies and three studies conducted in India and Pakistan, respectively. The populations represented by the included studies spanned all age groups and included participants primarily from hospital settings. As the data in this review were sourced from a variety of countries, age groups and settings, the heterogeneity was considerable and/or substantial for all
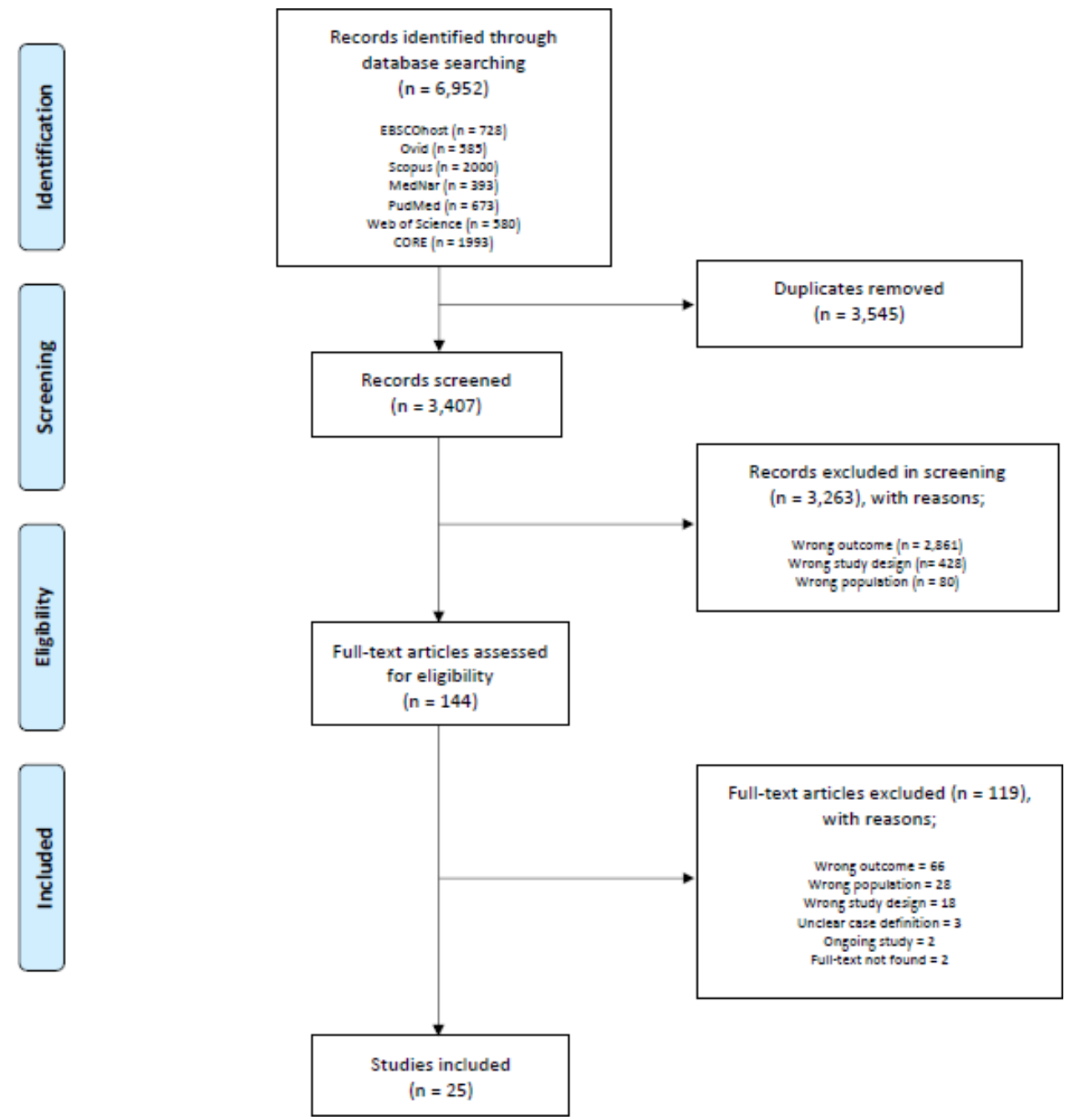

Figure 1 Flow diagram for selection of studies. 


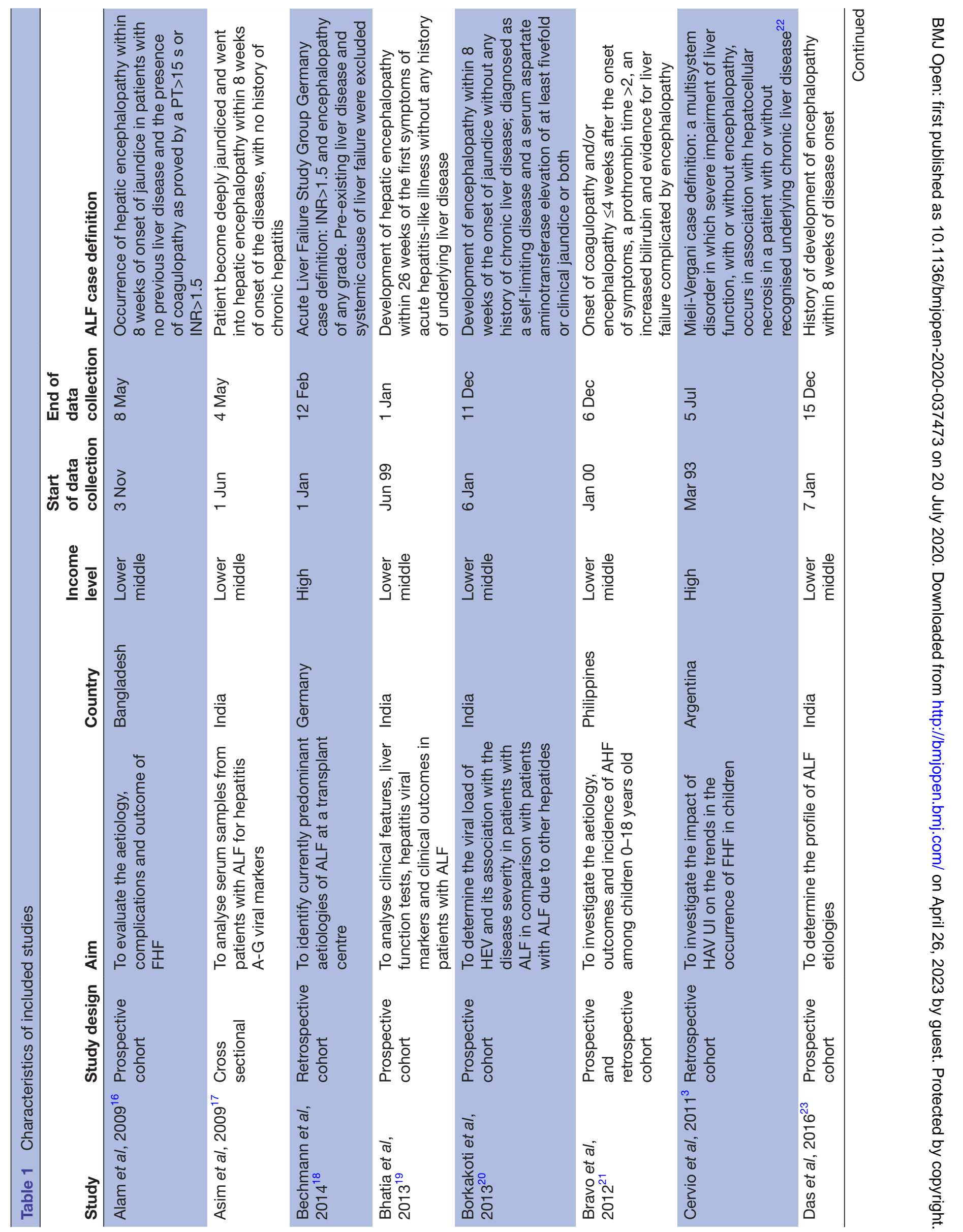




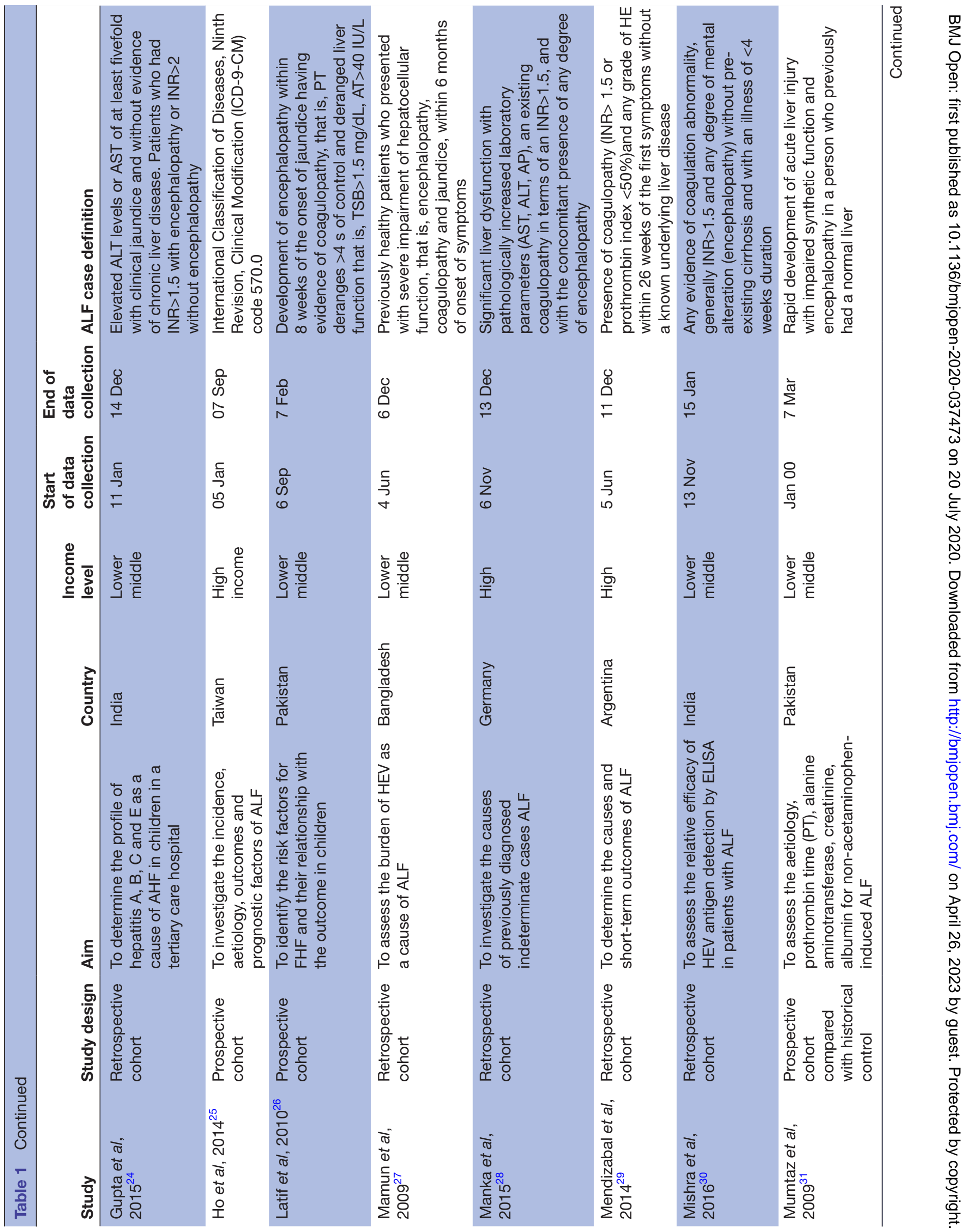




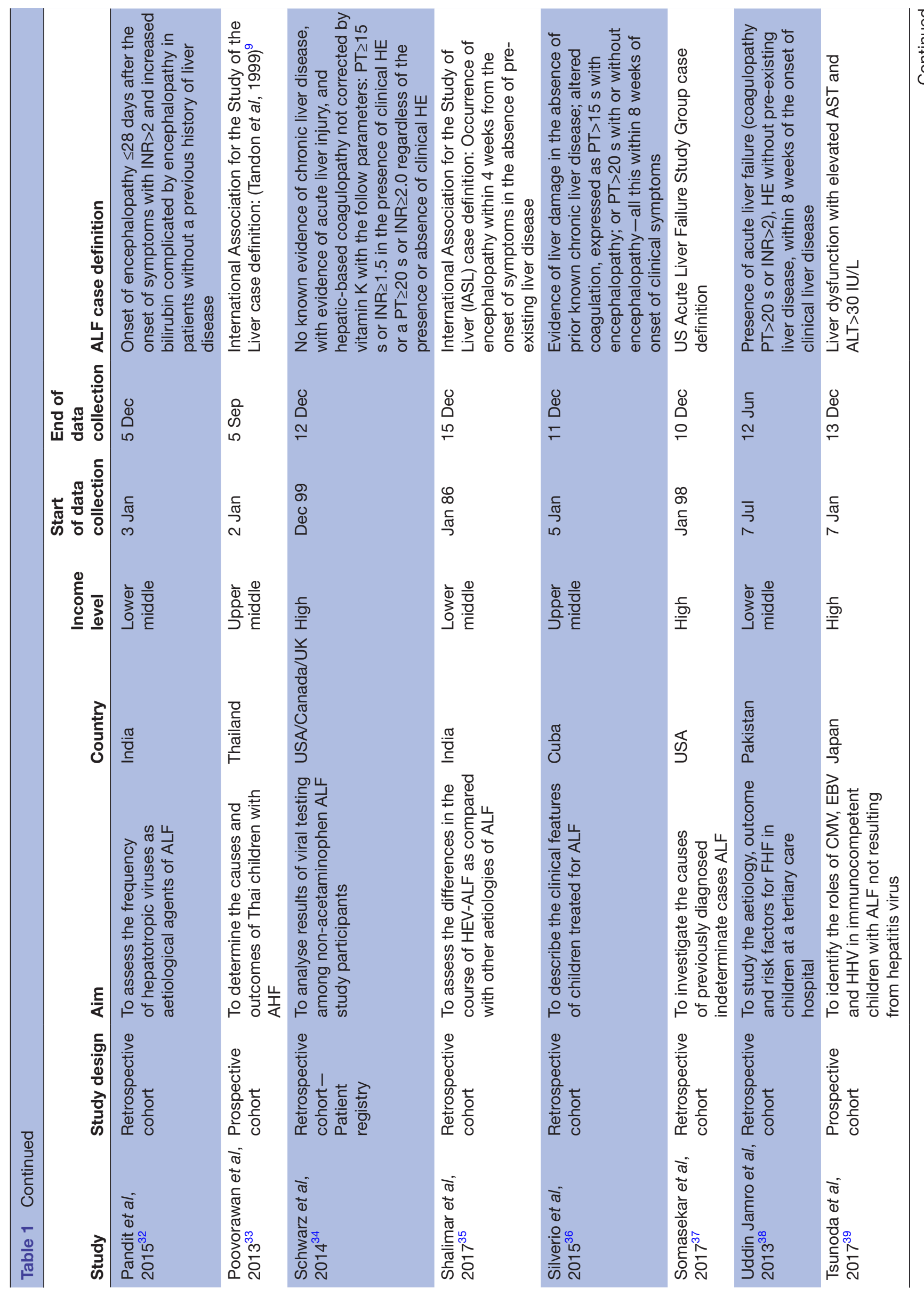

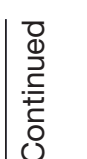


results. Thus, we narratively and graphically reported estimates of combined prevalence rates and the spreads of prevalence.

\section{Vaccine-preventable viral-induced ALF}

We narratively report the prevalence of HAV-induced and HBV-induced ALF by country immunisation status. The point prevalence of HAV-induced ALF in countries with no routine HAV immunisation at the time of data collection ranged from $2 \%$ to $81 \%$ with a combined rate of $27 \%$ (95\% CI 13\% to $43 \%$ ), while the prevalence in countries with routine HAV immunisation at the time of data collection ranged from $1 \%$ to $2 \%$ with a combined of rate of $2 \%$ (95\% CI $1 \%$ to $3 \%$ ) (figure 2). In Argentina, the prevalence of HAV-induced ALF prior to routine immunisation was approximately 50\% (95\% CI $45 \%$ to $55 \%$ ), compared with approximately $1 \%(95 \%$ CI $0 \%$ to $5 \%)$ after immunisation was introduced. The point prevalence of HBV-induced ALF in countries without universal HBV immunisation at the time of data collection ranged from $16 \%$ to $27 \%$ with a combined rate of $22 \%$ (95\% CI $16 \%$ to $30 \%$ ) (figure 3 ). The point prevalence of $\mathrm{HBV}$-induced ALF in countries with universal HBV immunisation at the time of data collection ranged from $0 \%$ to $83 \%$ with a combined rate of $20 \%$ (95\% CI $8 \%$ to $35 \%$ ).

\section{ALF attributable to non-vaccine-preventable viral infections}

The point prevalence of HCV-induced ALF ranged from $2 \%$ to $25 \%$ with a combined rate of $9 \%(95 \%$ CI $1 \%$ to $21 \%$ ) (online supplementary figure 1 ). The point prevalence of HEV-induced ALF ranged from $3 \%$ to $70 \%$ with a combined rate of $32 \%$ (95\% CI $24 \%$ to $41 \%$ ) (online supplementary figure 2). The point prevalence of HDV, $\mathrm{HHV} / \mathrm{HSV}$, CMV and EBV-induced ALF were estimated to have combined prevalences of $4 \%(95 \%$ CI $0 \%$ to $13 \%), 6 \%$ (95\% CI $1 \%$ to $12 \%), 13 \%$ (95\% CI $1 \%$ to $35 \%$ ) and $6 \%$ (95\% CI $0 \%$ to $24 \%), 10 \%$ (95\% CI $2 \%$ to $22 \%), 2 \%(95 \%$ CI $0 \%$ to $5 \%)$ and $1 \%$ (95\% CI $0 \%$ to $5 \%$ ), respectively (online supplementary figure 3 ). Data were not available to estimate the burden of ALF after infection with HDV, VZV, HPIVS, YFV, CA16 and/or HAdVs as outlined per the published protocol. ${ }^{10}$

\section{Outcomes of viral-induced ALF}

The narratively reported outcomes of viral-induced ALF were found to be severe. The mortality rates associated with viral-induced ALF in lower middle income countries ranged from $18 \%$ to $91 \%$ with a combined mortality rate of $50 \%$ (95\% CI 36\% to 64\%) (figure 4A). The mortality rates associated with viral-induced ALF in upper middle income countries ranged $3 \%$ to $45 \%$ with a combined mortality rate of $26 \%$ (95\% CI $1 \%$ to $63 \%$ ) (figure $4 \mathrm{~A}$ ). The mortality rates associated with viral-induced ALF in high-income countries ranged from $12 \%$ to $40 \%$ with a combined mortality rate of $29 \%$ (95\% CI $17 \%$ to $43 \%$ ) (figure 4A). The rate of encephalopathy associated with viral-induced ALF cases in children ranged from $69 \%$ to $100 \%$ with a combined rate of $89 \%(95 \%$ CI $79 \%$ to $97 \%)$ 


\begin{tabular}{|c|c|c|c|c|c|}
\hline Study & & Estimate $(95 \% \mathrm{Cl})$ & Country & Data start & Data end \\
\hline \multicolumn{6}{|l|}{ No routine vaccination } \\
\hline Asim et al., 2008 & - & $0.04(0.00,0.14)$ & India & Jun-01 & May-04 \\
\hline Mumtaz et al., 2009 & - & $0.02(0.00,0.08)$ & Pakistan & Jan-00 & Mar-07 \\
\hline Alam et al., 2009 & $=$ & $0.07(0.02,0.17)$ & Bangladesh & Nov-03 & May-08 \\
\hline Latif et al., 2010 & - & $0.56(0.41,0.70)$ & Pakistan & Sep-06 & Feb-07 \\
\hline Cervio et al., 2011 & $=$ & $0.50(0.45,0.55)$ & Argentina & Mar-93 & Jul-05 \\
\hline Bravo et al., 2012 & & $0.29(0.10,0.56)$ & Philippines & Jan-00 & Dec-06 \\
\hline Bhati et al., 2013 & & $0.52(0.31,0.72)$ & India & Jun-99 & Jan-01 \\
\hline Uddin Jamro et al., 2013 & $=$ & $0.81(0.69,0.90)$ & Pakistan & Jul-07 & Jun-12 \\
\hline Borkakoti et al., 2013 & $=$ & $0.07(0.05,0.11)$ & India & Jan-06 & Dec-11 \\
\hline Bechmann et al., 2014 & $=$ & $0.07(0.04,0.13)$ & Germany & Jan-01 & Feb-12 \\
\hline Gupta et al., 2015 & & $0.50(0.29,0.71)$ & India & Jan-11 & Dec-14 \\
\hline Pandit et al., 2015 & - & $0.66(0.49,0.80)$ & India & Jan-03 & Dec-05 \\
\hline Mishra et al., 2016 & $=$ & $0.22(0.10,0.39)$ & India & Nov-13 & Jan-15 \\
\hline Das et al., 2016 & - & $0.30(0.24,0.36)$ & India & Jan-07 & Dec-15 \\
\hline Shalimar et al., 2017 & 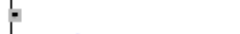 & $0.02(0.01,0.02)$ & India & Jan-86 & Dec-15 \\
\hline Subtotal $\left(\mathbb{F}^{2}=98.52 \%\right)$ & & $0.27(0.13,0.43)$ & & & \\
\hline \multicolumn{6}{|l|}{ Routine vaccination } \\
\hline Mendizabal et al., 2014 & - & $0.01(0.00,0.05)$ & Argentina & Jun-05 & Dec-11 \\
\hline Schwarz et al., 2014 & $=$ & $0.02(0.01,0.04)$ & USA/Canada/UK & Dec-99 & Dec-12 \\
\hline Somasekar et al., 2017 & $=$ & $0.02(0.01,0.05)$ & United States & Jan-98 & Dec-10 \\
\hline Subtotal $\left(I^{2}=N A\right)$ & & $0.02(0.01,0.03)$ & & & \\
\hline
\end{tabular}

Figure 2 Prevalence of HAV-induced ALF by country HAV immunisation status. ALF, acute liver failure; HAV, hepatitis A virus; $\mathrm{I}^{2}$, heterogeneity statistic.

(figure 4B). The need for liver transplantation with viralassociated ALF ranged from $4 \%$ to $62 \%$ with a combined rate of $25 \%$ (95\% CI $6 \%$ to $53 \%$ ) (figure $4 \mathrm{~B}$ ). The need for renal transplant in viral-associated ALF cases ranged from $4 \%$ to $34 \%$ with a combined rate of $18 \%(95 \%$ CI $2 \%$ to $43 \%$ ) (figure $4 \mathrm{~B}$ ).

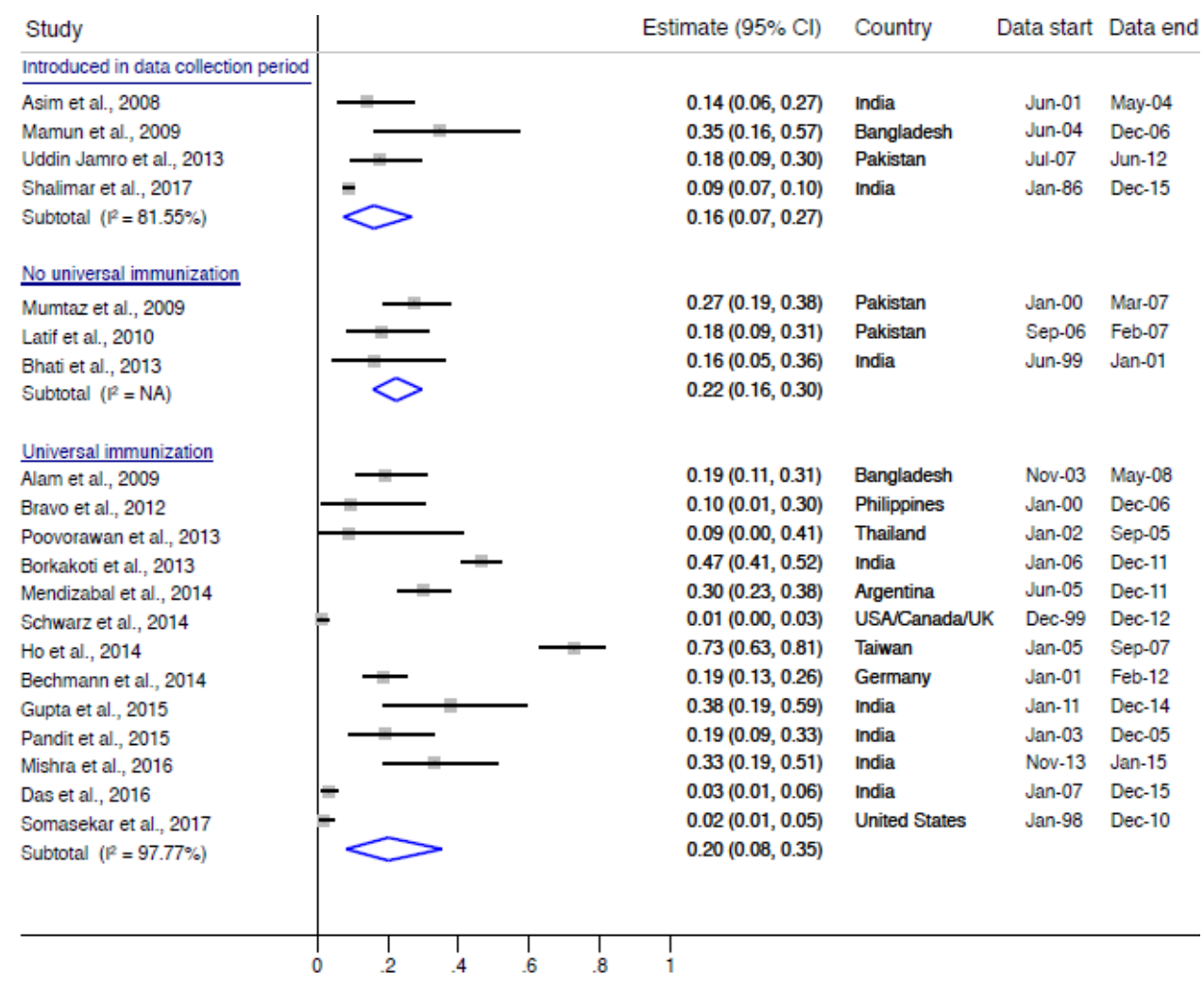

Figure 3 Prevalence of HBV-induced ALF by country HBV immunisation status. ALF, acute liver failure; HBV=hepatitis B virus; $\mathrm{I}^{2}$, heterogeneity statistic. 


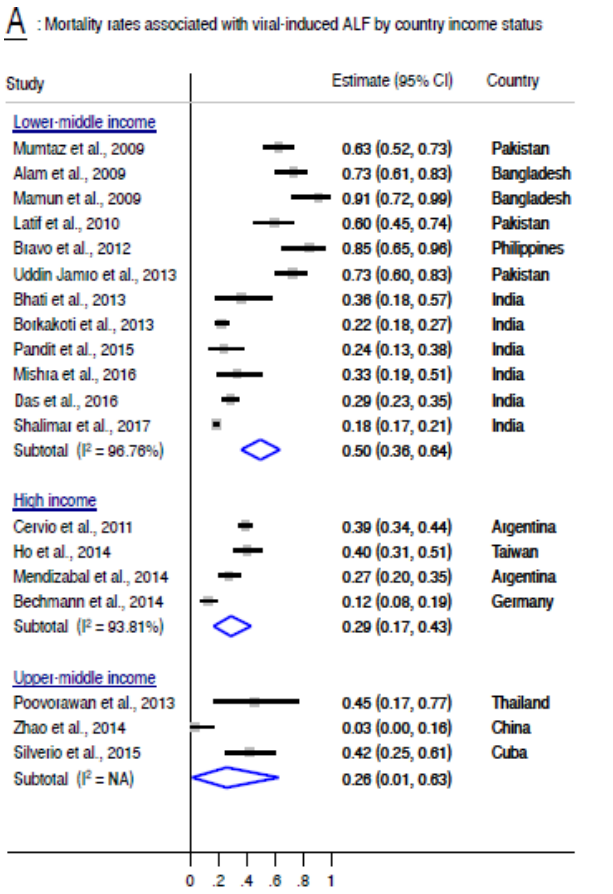

B : Prevalence of clinical ouncomes associated with vital-induces ALF

\begin{tabular}{|c|c|c|c|}
\hline Study & & Eetimate $(9 A \% \mathrm{Cl})$ & County \\
\hline \multicolumn{4}{|l|}{ Renal failure } \\
\hline Alam et al., 2009 & & $0.34(0.23,0.47)$ & Bangladesh \\
\hline Mumtaz etal., 2009 & - & $0.22(0.14,0.32)$ & Pakistan \\
\hline Shalmar et al., 2017 & 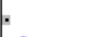 & $0.04(0.03,0.05)$ & Inda \\
\hline Subtotal $\left(R^{2}=\mathrm{NA}\right)$ & & $0.18(0.02,0.43)$ & \\
\hline \multicolumn{4}{|l|}{ Enceghalogathy } \\
\hline Laff et al., 2010 & & $-0.90(0.78,0.97)$ & Pakistan \\
\hline Cervio et al., 2011 & & $=\quad 0.83(0.79,0.87)$ & Argentina \\
\hline Bravo et al., 2012 & & - $\quad 0.69(0.48,0.86)$ & Philippines \\
\hline Uddin Jamro et al., 2013 & & $=1.00(0.94,1.00)$ & Pakistan \\
\hline Poovorawan et al, 2013 & & $-0.91(0.59,1.00)$ & Thailand \\
\hline Panditet al., 2015 & & $-0.89(0.77,0.96)$ & Inda \\
\hline Subtotal $\left({ }^{2}=85.11 \%\right)$ & & $\diamond 0.89(0.79,0.97)$ & \\
\hline \multicolumn{4}{|l|}{ Liver rransplant } \\
\hline Cervio et al., 2011 & $=$ & $0.62(0.56,0.67)$ & Argentina \\
\hline Mendizabal et al., 2014 & - & $0.54(0.46,0.62)$ & Argentina \\
\hline Bechmann et al, 2014 & $=$ & $0.12(0.07,0.18)$ & Germany \\
\hline Silverio et al., 2015 & - & $0.10(0.02,0.26)$ & Cuba \\
\hline Tsunoda et al., 2017 & - & $0.04(0.01,0.12)$ & Japan \\
\hline Subtotal $\left({ }^{2}=98.22 \%\right)$ & & $0.25(0.06,0.53)$ & \\
\hline
\end{tabular}

Figure 4 Prevalence of outcomes associated with viral-induced ALF. ALF, acute liver failure; $I^{2}$, heterogeneity statistic; NA, not applicable.

\section{Methodological quality}

Risk of bias scores were assigned by two reviewers (JP and $\mathrm{HSH})$ and are described in online supplementary table 1. Overall, a majority of the included studies were judged as having 'low risk' of bias. Only one included study was judged as having 'moderate risk' of bias due to lack of clarity around the representativeness of the study population to the national population, methods of participant selection and methods employed to reduce the likelihood of non-response.

\section{DISCUSSION}

This systematic review estimated the burden of ALF after infection with HAV, HBV, HCV, HEV, HSV/HHV, CMV, $\mathrm{EBV}$ and parvovirus B19. The prevalence of HAV-induced ALF is markedly lower in countries with routine HAV immunisation, while HEV was the most common aetiological cause of viral-induced ALF reported in this review. In addition, viral-induced ALF had poor outcomes as indicated by high fatality rates, which seem to increase with poor economic status of the studied countries.

The estimated prevalence of HAV-induced ALF in countries with routine HAV immunisation was markedly lower than the estimated prevalence in countries without routine HAV immunisation. When looking at countries with data before and after the introduction of routine HAV immunisation, the reduction of HAV-induced ALF due to vaccination is further highlighted. The combined prevalence of HBV-induced ALF was the same in settings with or without universal HBV immunisation. Countries without universal HBV immunisation programmes are likely to have weak healthcare systems; thus, the reported prevalence of HBV-induced ALF is assumed to be an underestimate of the true burden in these populations due to weak routine testing and reporting systems. Currently, there is one HEV vaccine (Hecolin) licensed in China that has shown promise with a high degree of efficacy in preventing HEV genotype IV infection in healthy individuals $16-65$ years. ${ }^{14}$ Further exploration of the efficacy of this vaccine for prevention of infection with genotypes I and II in different populations should be done to explore its application in different countries and HEV endemicity settings. ${ }^{15}$

This review estimated the mortality rate for viralinduced ALF to be approximately $50 \%$ in low-income and middle-income countries (LMICs) and less than $30 \%$ in upper-middle-income and high-income countries. Previous studies have estimated that mortality rates associated with ALF vary between $60 \%$ and $80 \%$, depending on the disease aetiology as well as a patient's access to care. Our review shows that viral-induced ALF still carries a significant mortality, though possibly lower than that reported for other ALF aetiologies. ${ }^{4}{ }^{5}$ Mortality data largely come from hospitals with the capacity to diagnose viral-induced ALF, thus deaths outside of the hospital system or ALF deaths without virological testing may not be captured in these mortality estimates. Liver transplantation is required by approximately $25 \%$ of viral-induced ALF cases and approximately $18 \%$ of viral-induced ALF cases required renal transplantation, globally. In addition to general lack of resources for transplantation, a significant proportion of potential candidates have contraindications to transplant related to poor socioeconomic status in LMICs. The transplant data included in this review 
may only reflect successful and unsuccessful transplants, not those that were needed but not carried out due to resource constraints or contraindications.

This review is limited by lack of data for some of the viral aetiologies of ALF including for VZV, HPIVs, YFV, CA16 and/or HAdVs, which may have led to an underestimation of the global burden of viral-induced ALF. Additionally, we believe that our findings underestimate the global burden of viral-induced ALF as some important causes of ALF (eg, HSV/HHV) are believed to be underrecognised as they require PCR testing for diagnosis. The included studies also used varying methods of virus detection including serology and molecular tests which further added to the heterogeneity in the results of our review. This is a well-recognised limitation in studies of ALF where diagnostics are often limited by cost in under-resourced regions where viral causes of ALF are more prevalent. The limited availability of data, including lack of same country data on burden of disease before and after introduction of immunisation, hindered most of the planned subgroup analyses outlined in the study protocol. Where data were available, high heterogeneity of the data led to planned meta-analyses and meta-regression analyses not being possible. Lastly, the diversity of viruses attributable to ALF cases led to low statistical power in meta-analyses conducted.

Future research should assess the burden of viralinduced ALF after infection with HDV, VZV, HPIVS, YFV, CA16 and HAdVs. Collectively, high-quality data on all viral aetiologies of ALF would allow for better pooling of results. The review team encourages future studies to incorporate health economic estimates and mathematical modelling where data permit to assist health policy decision-makers to better design strategies for the prevention and management of viral-induced ALF. Epidemiological-economic modelling of immunisation against HAV, HBV and HEV may well show that introduction of vaccination could lead to future cost savings in the long run due to prevented medical care and liver failure.

\section{CONCLUSIONS}

We successfully addressed the aim of the study, although data on VZV, HPIVs, YFV, CA16 and/or HAdVs were missing. Notwithstanding the noted limitations, it is clear that HAV, HBV and HEV - vaccine-preventable ALF aetiologies-account for a large proportion of ALF (approximately $21 \%, 20 \%, 32 \%$ of viral-induced ALF cases, respectively). The burden of ALF that is associated with vaccine-preventable ALF aetiologies should be used in conjunction with other available key evidence to inform practice and policies on immunisation, particularly in LMICs. A majority of LMICs have established universal vaccination against $\mathrm{HBV}$. The WHO has recently recommended the introduction of an HBV birth dose which is aimed at elimination of the virus and, if successful, will subsequently reduce the burden of HBV-induced ALF. Routine HAV immunisation in LMICs, however, is lacking.
More data are urgently needed to guide routine use of the vaccine in prevention of morbidity and mortality caused by the virus. Lastly, further applicability of HEV vaccines should be explored, especially in LMICs where resources for managing viral-induced ALF are glaringly lacking.

\section{Author affiliations}

${ }^{1}$ Vaccines for Africa Initiative, School of Public Health and Family Medicine, University of Cape Town Faculty of Health Sciences, Cape Town, South Africa ${ }^{2}$ Department of Statistical Sciences, University of Cape Town, Cape Town, South Africa

${ }^{3}$ Nuffield Department of Medicine, University of Oxford, Oxford, UK

${ }^{4}$ Department of Paediatrics, Red Cross War Memorial Children's Hospital, University of Cape Town, Cape Town, South Africa

${ }^{5}$ Department of Medicine, Division of Gastroenterology, Groote Schuur Hospital, University of Cape Town, Cape Town, South Africa

${ }^{6}$ Department of Medicine, Division of Hepatology, Groote Schuur Hospital, University of Cape Town, Cape Town, South Africa

${ }^{7}$ Institute of Infectious Disease and Molecular Medicine, University of Cape Town, South Africa, University of Cape Town Faculty of Health Sciences, Cape Town, South Africa

${ }^{8}$ Department of Pediatrics \& Child Health, Red Cross War Memorial Children's Hospital, University of Cape Town, Cape Town, South Africa

Contributors JP, GDH, BMK and RM conceived this study. JP implemented the review under the supervision of RM. JP and HSH performed the study search, screening and extraction of data under the guidance of RM. GDH and BMK provided methodological expertise for this review. SS, LG, MS, and WS provided content expertise for this review and all authors will provide comments on the final manuscript before publication. JP is the guarantor of this review.

Funding This research received no specific grant from any funding agency in the public, commercial or not-for-profit sectors. The Vaccines for Africa Initiative (VACFA) has funded the costs associated with the research and dissemination of the results, including publications.

Competing interests None declared.

Patient and public involvement Patients and/or the public were not involved in the design, or conduct, or reporting, or dissemination plans of this research.

Patient consent for publication Not required.

Provenance and peer review Not commissioned; externally peer reviewed.

Data availability statement Data are available in a public, open access repository. All data were taken from published articles available in the public domain.

Open access This is an open access article distributed in accordance with the Creative Commons Attribution Non Commercial (CC BY-NC 4.0) license, which permits others to distribute, remix, adapt, build upon this work non-commercially, and license their derivative works on different terms, provided the original work is properly cited, appropriate credit is given, any changes made indicated, and the use is non-commercial. See: http://creativecommons.org/licenses/by-nc/4.0/.

ORCID iD

Jenna Patterson http://orcid.org/0000-0002-3927-037X

\section{REFERENCES}

1 European Association for the Study of the Liver. Electronic address: easloffice@easloffice.eu, Clinical practice guidelines panel, Wendon, $\mathrm{J}$, et al. EASL clinical practical guidelines on the management of acute (fulminant) liver failure. J Hepatol 2017;66:1047-81.

2 Morabito V, Adebayo D. Fulminant hepatitis: definitions, causes and management. Health 2014;06:1038-48.

3 Cervio G, Trentadue J, D'Agostino D, et al. Decline in HAV-associated fulminant hepatic failure and liver transplant in children in Argentina after the introduction of a universal hepatitis: a vaccination program. Hepat Med 2011;3:99-106.

4 Bernal W, Auzinger G, Dhawan A, et al. Acute liver failure. Lancet 2010;376:190-201.

5 Wlodzimirow KA, Eslami S, Abu-Hanna A, et al. Systematic review: acute liver failure - one disease, more than 40 definitions. Aliment Pharmacol Ther 2012;35:1245-56. 
6 Lee WM, Stravitz RT, Larson AM. Introduction to the revised American association for the study of liver diseases position paper on acute liver failure 2011. Hepatology 2012;55:965-7.

7 Spearman CWN, McCulloch M, Millar AJW, et al. Liver transplantation at red cross war Memorial children's Hospital. S Afr Med J 2006;96:960-3.

8 O'Grady JG, failure Aliver. Acute liver failure. Postgrad Med J 2005;81:148-54.

9 Tandon BN, Bernauau J, O'Grady J, et al. Recommendations of the International association for the study of the liver Subcommittee on nomenclature of acute and subacute liver failure. $J$ Gastroenterol Hepatol 2002;14:403-4.

10 Patterson J, Hussey HS, Abdullahi LH, et al. The global epidemiology of viral-induced acute liver failure: a systematic review protocol. $B M J$ Open 2019;9:e029819.

11 Balk E, Ching M, Chen M, et al. Assessing the accuracy of Google translate to allow data extraction from trials published in non-English languages. Rockville, USA: Agency for Healthcare Research and Quality, 2013

12 Hoy D, Brooks P, Woolf A, et al. Assessing risk of bias in prevalence studies: modification of an existing tool and evidence of interrater agreement. J Clin Epidemiol 2012;65:934-9.

13 Werfalli M, Musekiwa A, Engel ME, et al. The prevalence of type 2 diabetes mellitus among older people in Africa: a systematic review study protocol. BMJ Open 2014;4:e004747.

14 Li S-W, Zhao Q, Wu T, et al. The development of a recombinant hepatitis E vaccine HEV 239. Hum Vaccin Immunother 2015;11:908-14.

15 Wu X, Chen P, Lin H, et al. Hepatitis E virus: current epidemiology and vaccine. Hum Vaccin Immunother 2016;12:2603-10.

16 Alam S, Azam G, Mustafa G, et al. Natural course of fulminant hepatic failure: the scenario in Bangladesh and the differences from the West. Saudi J Gastroenterol 2009;15:229-33.

17 Asim M, Singla R, Gupta RK, et al. Clinical \& molecular characterization of human TT virus in different liver diseases. Indian $J$ Med Res 2010;131:545-54

18 Bechmann LP, Manka P, Best J, et al. [Drug-induced liver injury as predominant cause of acute liver failure in a monocenter study]. Dtsch Med Wochenschr 2014;139:878-82.

19 Bhatia V, Dhawan A, Arora NK, et al. Urinary potassium loss in children with acute liver failure and acute viral hepatitis. $J$ Pediatr Gastroenterol Nutr 2013;57:102-8.

20 Borkakoti J, Hazam RK, Mohammad A, et al. Does high viral load of hepatitis $E$ virus influence the severity and prognosis of acute liver failure during pregnancy? J Med Virol 2013;85:620-6.

21 Bravo LC, Gregorio GV, Shafi F, et al. Etiology, incidence and outcomes of acute hepatic failure in 0-18 year old Filipino children. Southeast Asian J Trop Med Public Health 2012;43:764-72.

22 Dhawan A, Cheeseman P, Mieli-Vergani G. Approaches to acute liver failure in children. Pediatr Transplant 2004;8:584-8.

23 Das AK, Begum T, Kar P, et al. Profile of acute liver failure from north-east India and its differences from other parts of the country. Euroasian J Hepatogastroenterol 2016;6:111-5.
24 Gupta P, Mittal M, Bhat NK, et al. A hospital based retrospective study on hepatotropic viruses as a cause of acute viral hepatitis in children in Uttarakhand, India. Indian J Comm Health 2015;27:451-5.

25 Ho C-M, Lee C-H, Wang J-Y, et al. Nationwide longitudinal analysis of acute liver failure in Taiwan. Medicine 2014;93:e35.

26 Latif N, Mehmood K. Risk factors for fulminant hepatic failure and their relation with outcome in children. $J$ Pak Med Assoc 2010;60:175-8.

27 MamunAl M, Rahman S, Khan M, et al. HEV infection as an aetiologic factor for acute hepatitis: experience from a tertiary hospital in Bangladesh. J Health Popul Nutr 2009;27:14-19.

28 Manka P, Bechmann LP, Coombes JD, et al. Hepatitis E virus infection as a possible cause of acute liver failure in Europe. Clin Gastroenterol Hepatol 2015;13:1836-42.

29 Mendizabal M, Marciano S, Videla MG, et al. Changing etiologies and outcomes of acute liver failure: perspectives from 6 transplant centers in Argentina. Liver Transp/ 2014;20:483-9.

30 Mishra S, Borkakoti J, Kumar S, et al. Role of HEV antigen detection in HEV-related acute viral hepatitis and acute liver failure. $J$ Med Virol 2016;88:2179-85.

31 Mumtaz K, Azam Z, Hamid S, et al. Role of N-acetylcysteine in adults with non-acetaminophen-induced acute liver failure in a center without the facility of liver transplantation. Hepatol Int 2009;3:563-70.

32 Pandit A, Mathew LG, Bavdekar A, et al. Hepatotropic viruses as etiological agents of acute liver failure and related-outcomes among children in India: a retrospective hospital-based study. BMC Res Notes 2015;8:381.

33 Poovorawan $\mathrm{Y}$, Chongsrisawat V, Shafi F, et al. Acute hepatic failure among hospitalized Thai children. Southeast Asian J Trop Med Public Health 2013;44:50-3.

34 Schwarz KBO, Dell D, Lobritto SJ, et al. For the pediatric acute liver failure study, group. Analysis of viral testing in Nonacetaminophen pediatric acute liver failure. J Pediatr Gastroenterol Nutr 2014;59:616-23.

35 Kedia S, Gunjan D, Mahapatra SJ, et al. Acute liver failure due to hepatitis $E$ virus infection is associated with better survival than other etiologies in Indian patients. Dig Dis Sci 2017;62:1058-66.

36 Silverio $\mathrm{CE}$, Smithen-Romany $\mathrm{CY}$, Hondal $\mathrm{NI}$, et al. Acute liver failure in Cuban children. MEDICC Rev 2015;17:48-54.

37 Somasekar S, Lee D, Rule J, et al. Viral surveillance in serum samples from patients with acute liver failure by metagenomic nextgeneration sequencing. Clin Infect Dis 2017;65:1477-85.

38 Uddin Jamro BMC S, Mal Makheja P, Ahmed Soomro A. Etiology, outcome and risk factors for fulminant hepatic failure in children at a tertiary care Hospital, Sukkur, Pakistan. Rawal Medical Journal 2013;38:219-22.

39 Tsunoda T, Inui A, Iwasawa K, et al. Acute liver dysfunction not resulting from hepatitis virus in immunocompetent children. Pediatr Int 2017;59:551-6.

40 Zhao P, Wang C-Y, Liu W-W, et al. Acute liver failure in Chinese children: a multicenter investigation. Hepatobiliary Pancreat Dis Int 2014;13:276-80. 